\title{
SUPPLEMENTARY MATERIAL FOR ROLE OF NORMALIZATION IN SPECTRAL CLUSTERING FOR STOCHASTIC BLOCKMODELS
}

\author{
By Purnamrita Sarkar, Peter J. Bickel \\ University of California, Berkeley
}

\begin{abstract}
In this document we present technical details and accompanying lemmas which are necessary for the main results in the main manuscript [3]. When we make references to equations or theorems etc. in the main document, we follow the numbering scheme of the main document, and the references do not have any alphabets in them.
\end{abstract}

A. Proofs of Ancillary Concentration Results. In this section we state and prove some preliminaries that would be used throughout the rest of the supplementary material.

A.1. Generic concentration results. First we will present some concentration results involving functions of the degree of a node $d_{i}$. For simplicity, Lemmas A.1 to A.5 are proven for the Erdős-Rényi model with $p$ equal to a constant times $\rho_{n}$. However, an analogous proof holds even when $d_{i}$ is the degree of a node in a Stochastic Blockmodel, since $d_{i}$ is still a sum of $n-1$ independent Bernoulli's. The only difference is that $d_{0}$ becomes the conditional expectation of the degree under the Stochastic Blockmodel. Since we are allowing an uniform edge probability $\rho_{n}$, all the orders of magnitudes hold with $p$ replaced by $\rho_{n}$.

Lemma A.1. Let $d_{0}:=(n-1) p, \max _{i}\left|\left(\frac{d_{i}}{d_{0}}\right)^{-1 / 2}-1\right|=O_{P}\left(\sqrt{\frac{\log n}{n p}}\right)$

Proof. Let $U_{i}:=d_{i} / d_{0}$. Using a standard application of Chernoff bound we see that $P\left(\left|U_{i}-1\right| \geq \sqrt{\frac{6 \log n}{n p}}\right) \leq n^{-2}$. Let $\epsilon:=\sqrt{\frac{6 \log n}{n p}}$. First note that, $P\left[d_{i}=0\right]=(1-p)^{n-1}=O_{P}(\exp (-(n-1) p))$ and hence $U_{i}^{-1 / 2}$ is defined with probability converging to one exponentially fast.

For $x>0, x^{-1 / 2}$ is a continuous and monotonic function of $x$, and hence for $u:=(1-\epsilon)^{-1 / 2}$ and $\ell:=(1+\epsilon)^{-1 / 2}$, we have $P\left(U_{i} \in[\ell, u]\right) \geq 1-\delta$. Note that $(1-\epsilon)^{-1 / 2}=1+\epsilon / 2+3 \epsilon^{2} / 8\left(1-\epsilon_{0}\right)^{5 / 2}$ for some $\epsilon_{0} \in[0, \epsilon]$. For large enough $n$, the quadratic term can be made smaller than $\epsilon / 2$. Similarly, for large $n,(1+\epsilon)^{-1 / 2} \geq 1-\epsilon$. Thus, for large enough $n, P\left(U_{i}^{-1 / 2} \in[1-\epsilon, 1+\epsilon]\right) \geq$ 
$1-1 / n^{2}$. Finally,

$$
\begin{aligned}
P\left(\max _{i}\left|U_{i}^{-1 / 2}-1\right| \geq \epsilon\right) & =P\left(\exists i \in\{1, \ldots, n\},\left|U_{i}^{-1 / 2}-1\right| \geq \epsilon\right) \\
& \leq \sum_{i} P\left(\left|U_{i}^{-1 / 2}-1\right| \geq \epsilon\right) \leq 1 / n
\end{aligned}
$$

The following three lemmas can be proven in an analogous way.

LEMmA A.2. $\max _{i}\left|\left(\frac{d_{i}}{(n-1) p}\right)^{-3 / 2}-1\right|=O_{P}\left(\sqrt{\frac{\log n}{n p}}\right)$

Lemma A.3. Let $E:=\sum_{j} d_{j}, E_{0}:=n(n-1) p \cdot\left|\left(\frac{E}{E_{0}}\right)^{-3 / 2}-1\right|=O_{P}\left(\sqrt{\frac{\log n}{n^{2} \rho_{n}}}\right)$

LEMmA A.4. We have $\frac{1}{E} \sim \frac{1}{n(n-1) p}$.

Lemma A.5. Define $\bar{d}_{i}:=d_{i}-(n-1) p$, we have $\sum_{i} \bar{d}_{i}^{4}=O_{P}\left(n^{3} \rho_{n}^{2}\right)$.

Proof. We will bound the expectation and variance of $\sum_{i} \bar{d}_{i}^{4}$. Let $\bar{A}_{i j}:=$ $A_{i j}-p$, where $A$ is the adjacency matrix. By an application of the Rosenthal inequality, for some universal constant $C$ we have:

$$
E\left[\bar{d}_{i}^{4}\right]=E\left(\sum_{j} \bar{A}_{i j}\right)^{4} \leq C \max \left(\sum_{j} E \bar{A}_{i j}^{4},\left(\sum_{j} E \bar{A}_{i j}^{2}\right)^{2}\right)=O\left(n^{2} \rho_{n}^{2}\right)
$$

For the variance of $\bar{d}_{i}^{4}$, another application of the Rosenthal inequality with a different universal constant $C^{\prime}$ we have:

$$
\operatorname{var}\left(\bar{d}_{i}^{4}\right) \leq E\left[\bar{d}_{i}^{8}\right] \leq C^{\prime} \max \left(\sum_{j} E \bar{A}_{i j}^{8},\left(\sum_{j} E \bar{A}_{i j}^{2}\right)^{4}\right)=O\left(n \rho_{n}\right)^{4}
$$

Also, $\left|\operatorname{cov}\left[\bar{d}_{i}^{4}, \bar{d}_{k}^{4}\right]\right| \leq \operatorname{var}\left(\bar{d}_{i}^{4}\right)=O\left(n \rho_{n}\right)^{4}$. Thus $\operatorname{var}\left(\sum_{i} \bar{d}_{i}^{4}\right)=O\left(n^{6} \rho_{n}^{4}\right)$ and $E\left[\sum_{i} \bar{d}_{i}^{4}\right]=O\left(n^{3} \rho_{n}^{2}\right)$ leading to the result.

Proof of Lemma 5.5. First we prove Equation 5.3.

Proof of Equation 5.3. We see that $\operatorname{var}\left[\bar{d}_{i}^{(1)}\right]=(n \pi-1) \alpha_{n}\left(1-\alpha_{n}\right)$. Also $\operatorname{var}\left(\left(\bar{d}_{i}^{(1)}\right)^{2}\right) \leq E\left[\left(\bar{d}_{i}^{(1)}\right)^{4}\right]=O\left(n^{2} \rho_{n}^{2}\right)$, by an argument analogous to A.1. 
As for the covariance terms we have for $i \neq k \in C_{1}$,

$$
\begin{aligned}
E\left[\left(\bar{d}_{i}^{(1)}\right)^{2}\left(d_{k}^{(1)}\right)^{2}\right] & =E\left[\sum_{j_{1}, j_{2}, \ell_{1}, \ell_{2} \in C_{1}} \bar{A}_{i j_{1}} \bar{A}_{i j_{2}} \bar{A}_{k \ell_{1}} \bar{A}_{k \ell_{2}}\right]=\sum_{j_{1}, \ell_{1} \in C_{1}} E\left[\bar{A}_{i j_{1}}^{2}\right] E\left[\bar{A}_{k \ell_{1}}^{2}\right]+E\left[\bar{A}_{i k}^{4}\right] \\
& =\left(\sum_{j_{1} \in C_{1}} E\left[\bar{A}_{i j_{1}}^{2}\right]\right)^{2}+E\left[\bar{A}_{i k}^{4}\right]
\end{aligned}
$$

Hence $\operatorname{cov}\left(\left(\bar{d}_{i}^{(1)}\right)^{2},\left(d_{k}^{(1)}\right)^{2}\right)=E\left[\bar{A}_{i k}^{4}\right]$. Thus $\operatorname{var}\left(\sum_{i \in C_{1}} \bar{d}_{i}^{2}\right)=O_{P}\left(n^{3} \rho_{n}^{2}\right)$, and $\sum_{i \in C_{1}}\left(\bar{d}_{i}^{(1)}\right)^{2} \sim \sum_{i \in C_{1}} E\left[\left(\bar{d}_{i}^{(1)}\right)^{2}\right]$, proving the first result.

The second result is very similar, with the only difference that, $i \in C_{1}$ and we are dealing with $\sum_{j} \bar{A}_{i j}$ with $j \in C_{2}$. Thus, $E\left[\left(\bar{d}_{i}^{(2)}\right)^{2}\right]=n(1-\pi) \gamma_{n}\left(1-\gamma_{n}\right)$. As before $\operatorname{var}\left(\bar{d}_{i}^{(2)}\right)^{2}=O_{P}\left(n^{2} \rho_{n}^{2}\right)$. The difference is in the covariance terms; for $i \neq k \in C_{1}$ we see that $\left(\bar{d}_{i}^{(2)}\right)^{2}$ and $\left(d_{k}^{(2)}\right)^{2}$ are independent, since none of the edges considered in the two can be the same; this leads to zero covariance and gives the result.

Proof of Equation 5.4. We have:

$$
\sum_{i \in C_{1}}\left(x_{1} \bar{d}_{i}^{(1)}+y_{1} \bar{d}_{i}^{(2)}\right)^{2}=\sum_{i \in C_{1}}\left(x_{1}^{2}\left(\bar{d}_{i}^{(1)}\right)^{2}+y_{1}^{2}\left(\bar{d}_{i}^{(2)}\right)^{2}+2 x_{1} y_{1} \bar{d}_{i}^{(1)} \bar{d}_{i}^{(2)}\right)
$$

We will show that $\sum_{i \in C_{1}}\left(\bar{d}_{i}^{(1)}\right)^{2}$ and $\sum_{i \in C_{1}}\left(\bar{d}_{i}^{(1)}\right)^{2}$ are a larger order than $\sum_{i \in C_{1}} \bar{d}_{i}^{(1)} \bar{d}_{i}^{(2)}$.

Lemma 5.5 (Equation 5.3) shows that $\sum_{i \in C_{1}}\left(\bar{d}_{i}^{(1)}\right)^{2}$ is $C_{0} n^{2} \rho_{n}\left(1+o_{P}(1)\right)$. Using similar arguments as in Lemma 5.5, one can show that $\sum_{i \in C_{1}}\left(\bar{d}_{i}^{(1)}\right)^{2}$ is of the same order. Now, for $i \in C_{1}, E\left[\bar{d}_{i}^{(1)} \bar{d}_{i}^{(2)}\right]=0$, since $\bar{d}_{i}^{(1)}$ and $\bar{d}_{i}^{(2)}$ are independent and have zero expectation. As for $\operatorname{var}\left(\bar{d}_{i}^{(1)} \bar{d}_{i}^{(2)}\right)=E\left[\left(\bar{d}_{i}^{(1)}\right)^{2}\right] E\left[\left(\bar{d}_{i}^{(2)}\right)^{2}\right]=$ $O_{P}\left(n^{2} \rho_{n}^{2}\right)$. For $i \neq k \in C_{1}$,

$$
\operatorname{cov}\left(\bar{d}_{i}^{(1)} \bar{d}_{i}^{(2)}, \bar{d}_{k}^{(1)} \bar{d}_{k}^{(2)}\right)=\sum_{j_{1}, \ell_{1} \in C_{1}, j_{2}, \ell_{2} \in C_{2}} E\left[\bar{A}_{i j_{1}} \bar{A}_{i j_{2}} \bar{A}_{k \ell_{1}} \bar{A}_{k \ell_{2}}\right]=0
$$

The last step is true because $i \neq k \in C_{1}, j_{1}, \ell_{1} \in C_{1}$, whereas $j_{2}, \ell_{2} \in C_{2}$. Hence $\bar{A}_{i j_{2}}$ and $\bar{A}_{k \ell_{2}}$ will always be unpaired and hence contribute zero to the expectation. Thus $\sum_{i \in C_{1}} \operatorname{var}\left(\bar{d}_{i}^{(1)} \bar{d}_{i}^{(2)}\right)=O_{P}\left(n^{3} \rho_{n}^{2}\right)$. Hence $\sum_{i \in C_{1}} \bar{d}_{i}^{(1)} \bar{d}_{i}^{(2)}$ is of smaller order than $\sum_{i \in C_{1}}\left(\bar{d}_{i}^{(1)}\right)^{2}$ and $\sum_{i \in C_{1}}\left(\bar{d}_{i}^{(1)}\right)^{2}$, and proving our result. 
A.2. Concentration results for analysis of the unnormalized method. Here we present some definitions and preliminary results needed for proving the concentration result in Lemma 5.2, which is proved in Section E.

Let $Q:=\left[\begin{array}{ll}\mathbf{v}_{1} & \mathbf{v}_{2}\end{array}\right]$ be the matrix with column vectors as the eigenvectors of the population matrix. Also let $\widehat{Q}:=\left[\begin{array}{ll}\widehat{\mathbf{v}}_{1} & \widehat{\mathbf{v}}_{2}\end{array}\right]$ be the empirical version of $Q$. Let $C$ be a $2 \times 2$ matrix with $C=\widehat{Q}^{T} Q$. We write

$$
Q=\widehat{Q} C+R
$$

It is important to note that $\widehat{Q}^{T} R$ is the $2 \times 2$ zero matrix. First we prove that the norm of $R$ is small. Let $\widehat{\Lambda}$ be the $2 \times 2$ diagonal matrix with the top two eigenvalues (in magnitude) of $A$ along its diagonals. Left multiplying Equation (A.2) by $A$, and noting that $A \widehat{Q}=\widehat{Q} \widehat{\Lambda}$ we get:

$$
(A-P) Q=\widehat{Q} \widehat{\Lambda} C+A R-P Q=\widehat{Q}(\widehat{\Lambda} C-C \Lambda)+(A R-R \Lambda)
$$

By left multiplying each side of Equation A.2 with its transpose we also have

$$
C^{T} C=I_{2 \times 2}-R^{T} R
$$

Lemma A.6. Define a stochastic blockmodel (see Definition 2.2) with parameters $\left(\alpha_{n}, \beta_{n}, \gamma_{n}, \pi\right)$. Assume W.L.O.G that $\pi \alpha_{n}>(1-\pi) \beta_{n}$. We have

$$
\begin{aligned}
\left\|Q^{T}(A-P) Q\right\| & =O_{P}(1) \\
\left\|Q^{T}(A-P)^{2} Q\right\| & =O_{P}\left(n \rho_{n}\right) \\
\|R \Lambda\| & \geq \min \left(\left|\Lambda_{11}\right|,\left|\Lambda_{22}\right|\right)\|R\| / \sqrt{2} \\
\left\|R^{T} A R\right\| & \leq\|R\|^{2}\left|\widehat{\lambda}_{3}\right| \\
\left\|R^{T} A^{2} R\right\| & \leq\|R\|^{2} \widehat{\lambda}_{3}^{2}
\end{aligned}
$$

PROOF. In order to prove this lemma, we will first state a simple result which will be used heavily. Let $B$ be a $k \times k$ symmetric positive semi-definite matrix. Since the trace of a matrix equals the sum of its eigenvalues, we have the following inequality:

$$
\operatorname{trace}(B) / k \leq\|B\| \leq \operatorname{trace}(B)
$$

Now we present the proofs of Equations A.5 to A.9.

Proof of Equation A.5: . Let $B:=Q^{T}(A-P) Q$. We see that the entries of $B$ are of the form

$$
a \sum_{i \in C_{1}}\left(d_{i}^{(1)}-n \pi \alpha_{n}\right)+b \sum_{i \in C_{2}}\left(d_{i}^{(2)}-n(1-\pi) \beta_{n}\right)+c \sum_{i \in C_{1}}\left(d_{i}^{(2)}-n(1-\pi) \gamma_{n}\right) .
$$


For $B_{11}, a=x_{1}^{2}, b=y_{1}^{2}$ and $c=2 x_{1} y_{1}$; for $B_{22}, a=x_{2}^{2}, b=y_{2}^{2}$ and $c=2 x_{2} y_{2}$ and for $B_{12} a=x_{1} x_{2}, b=y_{1} y_{2}$, and $c=\left(x_{2} y_{1}+x_{1} y_{2}\right)$. Now $\sum_{i \in C_{1}}\left(d_{i}^{(1)}-\right.$ $\left.n \pi \alpha_{n}\right)$ is the sum of $\left(\begin{array}{c}n \pi \\ 2\end{array}\right)$ centered Bernoulli random variables, and hence is $O_{P}\left(n \sqrt{\rho_{n}}\right)$. Similarly, $\sum_{i \in C_{2}}\left(d_{i}^{(2)}-n(1-\pi) \beta_{n}\right)$ and $\sum_{i \in C_{1}}\left(d_{i}^{(2)}-n(1-\pi) \gamma_{n}\right)$ are also $O_{P}\left(n \sqrt{\rho_{n}}\right)$. From Lemma $5.1 x_{1}, x_{2}, y_{1}$ and $y_{2}$ are all of the form $C_{0} / \sqrt{n}$. Hence $B_{11}, B_{12}$ and $B_{22}$ are all $O_{P}(1)$. Hence $\|B\| \leq \sqrt{2} B_{\infty}=$ $O_{P}(1)$.

Proof of Equation A.6: . Let $B:=Q^{T}(A-P)^{2} Q$; we have:

$$
B_{11}=\left\|(A-P) v_{1}\right\|^{2} \quad B_{22}=\left\|(A-P) v_{2}\right\|^{2} \quad B_{12}=B_{21} \leq \sqrt{B_{11} B_{22}}
$$

Since $B$ is positive semi-definite, Equation A.10 gives $\|B\| \leq B_{11}+B_{22}$, where we have:

$$
\begin{aligned}
& B_{11}=\sum_{i \in C_{1}}\left(x_{1}\left(d_{i}^{(1)}-n \pi \alpha_{n}\right)+y_{1}\left(d_{i}^{(2)}-n(1-\pi) \gamma_{n}\right)\right)^{2}+\sum_{i \in C_{2}}\left(x_{1}\left(d_{i}^{(1)}-n \pi \gamma_{n}\right)+y_{1}\left(d_{i}^{(2)}-n(1-\pi) \beta_{n}\right)\right)^{2} \\
& B_{22}=\sum_{i \in C_{1}}\left(x_{2}\left(d_{i}^{(1)}-n \pi \alpha_{n}\right)+y_{2}\left(d_{i}^{(2)}-n(1-\pi) \gamma_{n}\right)\right)^{2}+\sum_{i \in C_{2}}\left(x_{2}\left(d_{i}^{(1)}-n \pi \gamma_{n}\right)+y_{2}\left(d_{i}^{(2)}-n(1-\pi) \beta_{n}\right)\right)^{2}
\end{aligned}
$$

Using Lemma 5.5 (Equation 5.4), and the fact that $x_{1}, x_{2}, y_{1}$ and $y_{2}$ are all constant factors of $1 / \sqrt{n}$ (Lemma 5.1), we see that $B_{11}$ and $B_{22}$ are $O_{P}\left(n \rho_{n}\right)$, and hence Equation (A.6) is proven.

Proof of Equation A.7. $\|R \Lambda\|=\sqrt{\left\|\Lambda R^{T} R \Lambda\right\|}$. Let $B:=\Lambda R^{T} R \Lambda$. Let $\lambda_{1}$ and $\lambda_{2}$ be the diagonal elements of $\Lambda$. Also WLOG let $\left|\lambda_{1}\right| \geq\left|\lambda_{2}\right|$. If the columns of $R$ are denoted by $r_{1}$ and $r_{2}$, then we have:

$$
B_{11}=\lambda_{1}^{2} r_{1}^{T} r_{1} \quad B_{12}=\lambda_{1} \lambda_{2} r_{1}^{T} r_{2} \quad B_{22}=\lambda_{2}^{2} r_{2}^{T} r_{2}
$$

Since $B$ and $R^{T} R$ are both positive semi-definite, from Equation A.10 we have, $\|B\| \geq\left(B_{11}+B_{22}\right) / 2 \geq \lambda_{2}^{2}\left(r_{1}^{T} r_{1}+r_{2}^{T} r_{2}\right) / 2$, and $\left\|R^{T} R\right\| \leq\left(r_{1}^{T} r_{1}+r_{2}^{T} r_{2}\right)$. Hence $\|R \Lambda\| \geq \sqrt{\lambda_{2}^{2}\left\|R^{T} R\right\| / 2}$, which gives us the result in Equation A.7.

Proof of Equation A.8. Let the eigenvalues of $A$ be ordered as $\left|\widehat{\lambda}_{1}\right| \geq$ $\left|\widehat{\lambda}_{2}\right| \geq \cdots \geq\left|\widehat{\lambda}_{n}\right|$. The adjacency matrix $A$ can be written as $\widehat{Q} \widehat{\Lambda} \widehat{Q}^{T}+$ $\widehat{Q^{\prime}} \widehat{\Lambda}^{\prime}{\widehat{Q^{\prime}}}^{T}$, where $\widehat{Q^{\prime}}$ is the $n \times n-2$ matrix with columns as eigenvectors corresponding to the eigenvalues $i>2 . \widehat{\Lambda}^{\prime}$ is the $n-2$ by $n-2$ diagonal matrices with eigenvalues $\widehat{\lambda}_{3}, \ldots, \widehat{\lambda}_{n}$ along the diagonal. We have $\left\|\widehat{\Lambda}^{\prime}\right\|=$ $\left\|\lambda_{3}\right\|$. Hence, $\left\|R^{T} A R\right\|=\left\|R^{T}{\widehat{Q^{\prime}}}^{\prime} \widehat{\Lambda}^{\prime} \widehat{Q}^{\prime} R\right\| \leq\|R\|^{2}\left|\widehat{\lambda}_{3}\right|$. 
Proof of Equation A.9. This is a simple consequence of the proof of Equation A.8.

\section{B. Bound on Misclassification error of Spectral Clustering using Oliveira's concentration result.}

Proof of Corollary 2.2. Theorem 2.1 shows that indeed $\|A-P\| \leq$ $4 \sqrt{\Delta \log (n / \delta)}$ for $A$ generated from a semi-sparse Stochastic Blockmodel. In fact, for $\gamma_{n}>0$, and $\alpha_{n} \beta_{n} \neq \gamma_{n}^{2}$, Lemma 5.1 shows that $\left|\lambda_{1}-\lambda_{2}\right|=C_{0} n \rho_{n}$. Let $x:=\left|\lambda_{1}-\lambda_{2}\right| / 4, a_{i}:=\lambda_{i}-2 x$, and $b_{i}:=\lambda_{i}+2 x$, for $i \in\{1,2\}$; there are no eigenvalues in $\left(a_{i}-x, a_{i}+x\right) \cup\left(b_{i}-x, b_{i}+x\right)$. Now, an application of Corollary 2.2 proves the result for eigenvectors of $A$.

In spirit of the analysis of misclassification error in [2], we construct a matrix $\Psi$ such that the $i^{\text {th }}$ row is the centroid of the cluster assigned to node $i$ (by the kmeans algorithm), also denoted by $\Psi_{i}$. Let $O$ denote a $2 \times 2$ diagonal matrix with $O_{i i}=\widehat{\mathbf{v}}_{i}^{T} \mathbf{v}_{i}$. Then a node $i$ is correctly classified if $\forall Q_{j} \neq Q_{i}(i$ and $j$ belong to two different population clusters), $\left\|\Psi_{i} O-Q_{j}\right\|>\left\|\Psi_{i} O-Q_{i}\right\|$.

When $i$ and $j$ belong to two different population clusters, we have $\| Q_{i}-$ $Q_{j} \|=\frac{1}{\sqrt{n \pi(1-\pi)}}:=s$. We will first show that $\left\|\Psi_{i} O-Q_{i}\right\|<s / 2$ is a sufficient condition for correct classification. So for $i$ and $j$ belonging to different population clusters, if $\left\|\Psi_{i} O-Q_{i}\right\|<s / 2$ we have:

$$
\left\|\Psi_{i} O-Q_{j}\right\| \geq\left\|Q_{i}-Q_{j}\right\|-\left\|\Psi_{i} O-Q_{i}\right\|>s / 2>\left\|\Psi_{i} O-Q_{i}\right\|,
$$

which implies that $i$ is correctly classified. Now let $\mathcal{M}:=\left\{i:\left\|\Psi_{i} O-Q_{i}\right\| \geq\right.$ $s / 2\}$. The set of misclassified nodes is a subset of this set. We have:

$$
|\mathcal{M}|=\sum_{i \in \mathcal{M}} 1 \leq \frac{4}{s^{2}} \sum_{i \in \mathcal{M}}\left\|\Psi_{i} O-Q_{i}\right\|^{2} \leq 4 n \pi(1-\pi)\left\|\Psi-Q O^{-1}\right\|_{F}^{2}\|O\|_{F}^{2}
$$

The kmeans algorithm minimizes $\|\Psi-\widehat{Q}\|_{F}$ over all $n \times k$ dimensional real matrices $\Psi$ with at most two unique rows, we have $\|\Psi-\widehat{Q}\|_{F} \leq\left\|Q O^{-1}-\widehat{Q}\right\|_{F}$, which also gives $\left\|\Psi-Q O^{-1}\right\|_{F} \leq 2\left\|\widehat{Q}-Q O^{-1}\right\|_{F}$. However Corollary 2.2 shows that, the operator norm $\left\|\mathbf{v}_{i} \mathbf{v}_{i}^{T}-\widehat{\mathbf{v}}_{i} \widehat{\mathbf{v}}_{i}^{T}\right\|=O_{P}\left(\sqrt{\log n / n \rho_{n}}\right)$. Now, $\left\|\mathbf{v}_{i}-\left(\mathbf{v}_{i}^{T} \widehat{\mathbf{v}}_{i}\right) \widehat{\mathbf{v}}_{i}\right\|=\left\|\left(\mathbf{v}_{i} \mathbf{v}_{i}^{T}-\widehat{\mathbf{v}}_{i} \widehat{\mathbf{v}}_{i}^{T}\right) \mathbf{v}_{i}\right\|=O_{P}\left(\sqrt{\log n / n \rho_{n}}\right)$ for $i \in\{1,2\}$; hence $\left\|\widehat{Q}-Q O^{-1}\right\|_{F}=O_{P}\left(\sqrt{\log n / n \rho_{n}}\right)$. Moreover, $\left|1-\left(\mathbf{v}_{i}^{T} \widehat{\mathbf{v}}_{i}\right)^{2}\right|=\mid \mathbf{v}_{i}^{T}\left(\mathbf{v}_{i}-\right.$ $\left.\left(\mathbf{v}_{i}^{T} \widehat{\mathbf{v}}_{i}\right) \widehat{\mathbf{v}}_{i}\right) \mid=O_{P}\left(\sqrt{\log n / n \rho_{n}}\right)$, giving $\|O\|_{F}=1+o_{P}(1)$. Hence we have, $|\mathcal{M}| / n=O_{P}\left(\log n / n \rho_{n}\right)$, which upper bounds the fraction of misclassified nodes. In order to analyze misclassification error of $\widetilde{A}$, one can use Lemma 6.1 and Corollary 2.2 to first show that the eigenvectors of $\widetilde{A}$ converge in the appropriate sense. Then, an identical argument yields the same rate for $\widetilde{A}$. 
C. Analysis of the limiting ratio $\widetilde{d}_{11}^{2} / \widehat{d}_{11}^{2}$ in the simple blockmodel with two equal sized classes and $\alpha_{n}=\beta_{n}$.

Proof of Corollary 3.2. Consider the Stochastic Blockmodel with two equal sized classes, and $\alpha_{n}=\beta_{n}$. The population eigenvalues and eigenvectors take the following simple form.

$$
\begin{array}{ll}
x_{1}=y_{1}=1 / \sqrt{n} & x_{2}=-y_{2}=1 / \sqrt{n} \\
\lambda_{1}=\frac{\left(\alpha_{n}+\gamma_{n}\right) n}{2} & \lambda_{2}=\frac{\left(\alpha_{n}-\gamma_{n}\right) n}{2}
\end{array}
$$

Substituting these in Equation 3.1 we obtain the following:

$$
\widehat{d}_{11}^{2} \sim \frac{4}{n^{2}} \frac{\alpha_{n}^{2}+\gamma_{n}^{2}}{\left(\alpha_{n}^{2}-\gamma_{n}^{2}\right)^{2}}\left(\alpha_{n}\left(1-\alpha_{n}\right)+\gamma_{n}\left(1-\gamma_{n}\right)\right)
$$

For the normalized method, substituting $\mu_{1}=\mu_{2}=\mu=\left(\alpha_{n}+\gamma_{n}\right) / 2$ and $\nu_{2}=1-\gamma_{n} / \mu$ in Equation 3.3 we get:

$$
\widetilde{d}_{11}^{2} \sim \frac{1}{n^{2} \mu^{2}}\left(\frac{\alpha_{n}\left(1-\alpha_{n}\right)+\gamma_{n}\left(1-\gamma_{n}\right)}{4}+\frac{\alpha_{n} \gamma_{n}(1-\mu)}{\mu \nu_{2}^{2}}\right)
$$

Let $x:=\gamma_{n} / \alpha_{n}$. Now the ratio of the above limiting expressions, denoted by $f_{n}\left(\alpha_{n}, x\right)$, can be written as:

$$
f_{n}\left(\alpha_{n}, x\right)=1 / 4+\frac{x}{2\left(x^{2}+1\right)}\left(\frac{4\left(1-\alpha_{n}(1+x) / 2\right)(1+x)}{\left(1-\alpha_{n}\right)+x\left(1-\alpha_{n} x\right)}-1\right)
$$

In the semi-sparse regime, $\alpha_{n}, \gamma_{n} \rightarrow 0$, and hence $\frac{\left(1-\alpha_{n}(1+x) / 2\right)(1+x)}{\left(1-\alpha_{n}\right)+x\left(1-\alpha_{n} x\right)} \rightarrow 1$, giving the required result. Since $2 x \leq x^{2}+1$, the limit never exceeds one.

Dense regime. We will break up the parameter space in the following parts. First consider $x>1$. We have:

$$
\frac{1-\alpha_{n}(1+x) / 2}{1-\alpha_{n}+x\left(1-\alpha_{n} x\right)}=1 / 2+\frac{\alpha_{n}(1-x)\left(1 / \alpha_{n}-x\right)}{1-\alpha_{n}+x\left(1-\alpha_{n} x\right)}
$$

Since $x \leq 1 / \alpha_{n}$, the above expression would always be smaller than a half when $x>1$. Hence, Equation C.3 translates to the following after some simple algebra:

$$
f_{n}\left(\alpha_{n}, x\right) \leq 1 / 4+\frac{x(1+2 x)}{2\left(x^{2}+1\right)} \leq 1.31
$$


Now we take $x \leq 1$, and further break up this regime into $\alpha_{n} \leq 1 / 2$ and $\alpha_{n}>1 / 2$. For $x \leq 1, \alpha_{n} \leq 1 / 2$, we have:

$$
f_{n}\left(\alpha_{n}, x\right) \leq 1 / 4+\frac{3-\alpha_{n}-2 \alpha_{n} x}{1-\alpha_{n}} \frac{x}{2\left(1+x^{2}\right)} \leq 1 / 4+\frac{x(2.5-x)}{1+x^{2}} \leq 1
$$

The first inequality uses $1-\alpha_{n}+x\left(1-\alpha_{n} x\right) \geq\left(1-\alpha_{n}\right)(1+x)$. The second inequality uses the fact the function is increasing in $\alpha_{n}$ and is thus maximized at $\alpha_{n}=1 / 2$.

Finally for the $x<1, \alpha_{n}>1 / 2$ case, we note that $\left(1-\alpha_{n}(1+x) / 2\right) /(1-$ $\left.\alpha_{n}+x\left(1-\alpha_{n} x\right)\right)$ is increasing in $\alpha_{n}$, and is therefore maximized at $\alpha_{n}=1$. This gives us the following upper bound, which can again be bounded by 1.31 by simple algebra.

$$
f_{n}\left(\alpha_{n}, x\right) \leq 1 / 4+\frac{2+x}{2\left(1+x^{2}\right)} \leq 1.31
$$

\section{Analysis of the normalized method in the zero communica-}

tion case. In this section we prove some results in Section 4, which were omitted from the main document for ease of exposition.

Proof of Lemma 4.2. We have, $x_{n}(i)-\sum_{i} x_{n}(i) / n=y_{n}(i)+z_{n}(i)$, where $\mathbf{y}_{n}$ and $\mathbf{z}_{n}$ are the centered $\mathbf{x}_{n}^{1}$ and $\mathbf{R}_{n}$ vectors respectively. Now, $\left\|\mathbf{z}_{n}\right\|^{2} \leq\left\|\mathbf{R}_{n}\right\|^{2}=o_{P}\left(\left\|\mathbf{x}_{n}^{1}\right\|^{2}\right)$ and $\left\|\mathbf{y}_{n}\right\|^{2}=\left\|\mathbf{x}_{n}^{1}\right\|^{2}\left(1+o_{P}(1)\right)$ by assumption $\left|\sum_{i} x_{n}^{1}(i) / n\right|=o_{P}\left(\left\|\mathbf{x}_{n}^{1}\right\| / \sqrt{n}\right)$. Hence, $\left\|\mathbf{z}_{n}\right\|=o_{P}\left(\left\|\mathbf{y}_{n}\right\|\right)$ and an application of the Cauchy-Schwarz inequality yields the result.

Proof OF Lemma 4.3. We will use the fact that $\|\widetilde{\mathbf{r}}\|^{2}=1-\widetilde{c}_{11}^{2}=$ $\sum_{i}\left(\widetilde{\mathbf{u}}_{i}-\sum_{i} \widetilde{\mathbf{u}}_{i} / n\right)^{2}$. Since one can explicitly obtain the expression of $u_{i}$, the basic idea is to use term by term Taylor approximation to obtain the norm of the residual. However, since the sum is over $n$ elements with $n \rightarrow \infty$, one needs to bound the elements uniformly over $n$.

It is easy to check that the vector $\left\langle\sqrt{d_{i} / E}\right\rangle$ is an eigenvector of $\widetilde{A}$ with eigenvalue one. Assumption 2.1 guarantees that $A$ is connected with high probability, and so the principal eigenvalue has multiplicity one. Thus $\mathbf{u}_{i}=$ $\sqrt{d_{i} / E}$. We have

$$
\sqrt{x}=\sqrt{x_{0}}+\frac{x-x_{0}}{2 \sqrt{x_{0}}}(1+S) \quad \frac{1}{\sqrt{y}}=\frac{1}{\sqrt{y_{0}}}-\frac{y-y_{0}}{2 y_{0}^{3 / 2}}\left(1+S^{\prime}\right) .
$$


where $S=\sqrt{x_{0}} \int_{0}^{1}\left(\left(x_{0}+\tau\left(x-x_{0}\right)\right)^{-1 / 2}-x_{0}^{-1 / 2}\right) d \tau$. For $\tau \in[0,1], \mid\left(x_{0}+\right.$ $\left.\tau\left(x-x_{0}\right)\right)^{-1 / 2}-x_{0}^{-1 / 2}|\leq| x^{-1 / 2}-x_{0}^{-1 / 2} \mid$, and hence $|S| \leq\left|\left(x_{0} / x\right)^{1 / 2}-1\right|$. Similarly $\left|S^{\prime}\right| \leq\left|\left(E_{0} / E\right)^{3 / 2}-1\right|=O_{P}\left(\sqrt{\log n / n^{2} \rho_{n}}\right)$ applying the Chernoff bound (Lemma A.3). Setting $x=d_{i}, x_{0}=d_{0}$, where $d_{0}:=(n-1) p y=$ $E, y_{0}=E_{0}$ where $E_{0}:=n(n-1) p$ we get:

$$
\begin{aligned}
& \widetilde{u}_{i}=\frac{\sqrt{d_{0}}}{\sqrt{E_{0}}}+\frac{\bar{d}_{i}}{2 \sqrt{d_{0} E_{0}}}+R \\
& R=\frac{\bar{d}_{i}}{2 \sqrt{d_{0} E_{0}}} S_{i}-\frac{E-E_{0}}{2} \sqrt{\frac{d_{0}}{E_{0}^{3}}}\left(1+S^{\prime}\right)-\frac{E-E_{0}}{2 \sqrt{d_{0} E_{0}^{3}}} \bar{d}_{i}\left(1+S_{i}\right)\left(1+S^{\prime}\right) \\
& \left|S^{\prime}\right|=o_{P}(1), \quad \quad \max _{i}\left|S_{i}\right|=o_{P}(1)
\end{aligned}
$$

We will now invoke Lemma 4.2 with $\mathbf{x}_{n}$ as $\widetilde{\mathbf{u}}$. Let $\mathbf{c}_{n}$ be the vector of constants $\sqrt{d_{0} / E_{0}}$, and $x_{n}^{1}(i):=\frac{\bar{d}_{i}}{2 \sqrt{d_{0} E_{0}}}$. Hence $\left\|\mathbf{x}_{n}^{1}\right\| \sim C_{0} / \sqrt{n \rho_{n}}$, and the average $\sum_{i} x_{n}^{1}(i) / n$ is $O_{P}\left(1 / \sqrt{n^{3} \rho_{n}}\right)=o_{P}\left(\left\|\mathbf{x}_{n}^{1}\right\| / \sqrt{n}\right)$. Now using Lemmas A.1, A.3 and A.4 we can show that $\|R\|=o_{P}\left(\left\|\mathbf{x}_{n}^{1}\right\|\right)$. Lemma 4.2 now gives the result.

E. Supplementary Material for Analysis of the unnormalized method. Let $A$ be generated from a $\left(\alpha_{n}, \beta_{n}, \gamma_{n}, Z\right)$ blockmodel defined in Definition 2.2. In this section we will prove the lemmas on deviation of principal eigenvalues and eigenvectors presented in Section 5.

E.1. Population eigenvalues and eigenvectors. First we prove properties of the population eigenvalues and eigenvectors via Lemma 5.1.

Proof of Lemma 5.1. Let the eigenvalues of $P$ ordered in decreasing order of absolute value be $\lambda_{1}, \lambda_{2}$, etc.

Trailing eigenvalues of $P$ : We will first show that $\lambda_{1}, \lambda_{2}$ are $O\left(n \rho_{n}\right)$, whereas $\lambda_{i}=O\left(\rho_{n}\right)$ for $i>2$. Consider the diagonal matrix $P_{D}$ with first $n \pi$ elements along the diagonal as $-\alpha_{n}$ and remaining $n(1-\pi)$ elements as $-\beta_{n}$. When $\alpha_{n} \beta_{n} \neq \gamma_{n}^{2}, P_{B}:=P-P_{D}$ is a rank two blockwise constant matrix, whose two nonzero eigenvalues are $O\left(n \rho_{n}\right)$ in magnitude. Also, $\left\|P-P_{B}\right\|=O\left(\rho_{n}\right)$. Hence, Weyl's inequality shows that the largest two eigenvalues of $P$ are also $O\left(n \rho_{n}\right)$, while the rest are all $O\left(\rho_{n}\right)$.

Principal eigenvalues and eigenvectors of $P$ : It is easy to show that the principal eigenvectors of $P$ are blockwise constant. Let $x$ and $y$ be $v(i)$ for $i \in C_{1}$ and $i \in C_{2}$ respectively. The coefficients $(x, y, \lambda)$ satisfy the following set of equations:

$$
\left(n \pi \alpha_{n}-\alpha_{n}\right) x+n(1-\pi) \gamma_{n} y=\lambda x ; \quad n \pi \gamma_{n} x+\left(n(1-\pi) \beta_{n}-\beta_{n}\right) y=\lambda y
$$


If $x$ or $y$ is zero, then $\gamma_{n}=0$, and we get the zero communication setting. When $\gamma_{n}>0, x, y$ are nonzero and writing $\xi=x / y$ and eliminating $\lambda$ gives the following quadratic equation.

$$
\pi \gamma_{n} \xi^{2}-\left(\pi \alpha_{n}-(1-\pi) \beta_{n}-\left(\alpha_{n}-\beta_{n}\right) / n\right) \xi-(1-\pi) \gamma_{n}=0
$$

Also, we have $\lambda=n\left(\pi \gamma_{n} \xi+(1-\pi) \beta_{n}-\beta_{n} / n\right)$. If $\alpha_{n} \beta_{n}=\gamma_{n}^{2}$, then some algebra shows that $\lambda_{2}=O_{P}(1)$; since this is not of the form $C_{0} n \rho_{n}$, our subsequent results will not hold. An example of this is the case where $\alpha_{n}=$ $\beta_{n}=\gamma_{n}$, because then we just have a Erdős-Rényi graph on hand.

For $i \in\{1,2\}, x_{i}=\frac{\xi_{i}}{\sqrt{n\left(\pi \xi_{i}^{2}+(1-\pi)\right)}}$ and $y_{i}=\frac{1}{\sqrt{n\left(\pi \xi_{i}^{2}+(1-\pi)\right)}}$. Some algebraic manipulation gives the first two expressions in Equation 5.1. From Equation E.1, we also have:

$$
\pi \gamma_{n} x^{2}-\left(\pi \alpha_{n}-(1-\pi) \beta_{n}-\left(\alpha_{n}-\beta_{n}\right) / n\right) x y-(1-\pi) \gamma_{n} y^{2}=0,
$$

which gives $x_{1} y_{1}+x_{2} y_{2}=\left(\pi\left(x_{1}^{2}+x_{2}^{2}\right)-(1-\pi)\left(y_{1}^{2}+y_{2}^{2}\right)\right) \frac{\gamma_{n}}{\pi \alpha_{n}-(1-\pi) \beta_{n}}=0$.

E.2. Concentration of empirical principal eigenvalues. Now we will move on to the sharp deviation results of the principal eigenvalues of $A$ in the semisparse regime. Theorem 2.1 ([1]) translates to the following:

Corollary E.1. Define a semi-sparse stochastic blockmodel (see Definition 2.2) with parameters $\left(\alpha_{n}, \beta_{n}, \gamma_{n}, \pi\right)$. Assume W.L.O.G that $\pi \alpha_{n}>$ $(1-\pi) \beta_{n}$. Let $A$ be the adjacency matrix of a $n$ node graph generated from this model. Let $\widehat{\lambda}_{1}, \widehat{\lambda}_{2}$ and $\widehat{\lambda}_{3}$ be the first, second and third largest eigenvalues (in magnitude) of A. Also, let $\lambda_{1}$ and $\lambda_{2}$ be the population versions respectively. For the principal eigenvalues, we have, for $i \in\{1,2\}$, $\widehat{\lambda_{i}}=\lambda_{i}\left(1+O_{P}\left(\sqrt{\log n / n \rho_{n}}\right)\right.$. For $\widehat{\lambda}_{3}$ we have:

$$
\widehat{\lambda}_{3}=O_{P}\left(\sqrt{n \rho_{n} \log n}\right) .
$$

PROOF. The first result is a straightforward application of Theorem 2.1, since $d, \Delta$ and $\lambda_{i}, i \in\{1,2\}$ (see Lemma 5.1) are all of the form $C_{0} n \rho_{n}$. Also Assumption 2.1 guarantees the condition on $\Delta$.

For the second result, let us consider the eigenvalues of $A$ in decreasing order, i.e. $\lambda_{1}(A) \geq \lambda_{2}(A) \geq \ldots$ etc. By the Perron Frobenius theorem we know that $\hat{\lambda}_{1}=\lambda_{1}(A)$. Let $\widehat{\lambda}_{2}=\left|\lambda_{k}(A)\right|$, where $k \in\{2, n\}$. We have:

$$
\begin{aligned}
\hat{\lambda}_{3}=\max _{i \notin\{1, k\}}\left|\lambda_{i}(A)\right| & \leq \max _{i \notin\{1, k\}}\left(\left|\lambda_{i}(A)-\lambda_{i}(P)\right|+\left|\lambda_{i}(P)\right|\right) \\
& \leq \max _{i \notin\{1, k\}}\left|\lambda_{i}(A)-\lambda_{i}(P)\right|+\max _{i \notin\{1, k\}}\left|\lambda_{i}(P)\right| \\
& =O_{P}\left(\sqrt{n \rho_{n} \log n}\right)
\end{aligned}
$$


The last step uses Weyl's inequality and the fact that $\max _{i \notin\{1, k\}}\left|\lambda_{i}(P)\right|=$ $O_{P}\left(\rho_{n}\right)$.

Now we present Lemmas E.1, E.2 and E.3 which will be instrumental in proving our sharp deviation bound on the principal empirical eigenvalues of $A$.

Lemma E.1. Let $C$ be defined as in Equation A.2. We have $\| C C^{T}-$ $I_{2 \times 2} \|=O_{p}\left(1 / n \rho_{n}\right)$.

Proof. Equation A.4 gives $C^{T} C=I_{2 \times 2}-R^{T} R$. The eigenvalues of $C C^{T}$ and $C^{T} C$ are the same; let us denote them by $c_{1}$ and $c_{2}\left(\left|c_{1}\right| \geq\left|c_{2}\right|\right)$. Since $\left\|R^{T} R\right\|=O_{p}\left(1 / n \rho_{n}\right)$ (Lemma E.3), Weyl's inequality yields $c_{i} \leq 1 \leq c_{i}+$ $\left\|R^{T} R\right\|$, i.e. $c_{i} \in\left(1-O_{p}\left(1 / n(1-\pi) \beta_{n}\right), 1\right)$, thus proving the result.

Lemma E.2. Let $C$ be defined as in Equation A.2. For a real diagonal $2 \times 2$ matrix $L$, let $\Gamma$ be the $2 \times 2$ matrix of eigenvalues of $C^{T} L C$. If $L_{i i}, i \in$ $\{1,2\}$ and $\left|L_{11}-L_{22}\right|$ are all of the same order, we have:

$$
\Gamma_{i i}=L_{i i}\left(1+O_{p}\left(\frac{1}{n \rho_{n}}\right)\right) \quad \text { For } i \in\{1,2\}
$$

Proof. Let $\operatorname{det}($.$) be the determinant of the square matrix (.), and let$ trace(.) denote the trace of the square matrix (.). The product of the eigenvalues of $C^{T} L C$ is given by $\Gamma_{11} \Gamma_{22}=\operatorname{det}\left(C^{T} L C\right)$. The sum of the eigenvalues is given by $\Gamma_{11}+\Gamma_{22}=\operatorname{trace}\left(C^{T} L C\right)$. Let $M:=C C^{T}-I_{2 \times 2}$. From Lemma E.1 we have $\|M\|=O_{p}\left(1 / n \rho_{n}\right)$. Every element of $M$ is also $O_{p}\left(1 / n \rho_{n}\right)$ (using $\left.\left|M_{i j}\right| \leq\|M\|_{F} \leq \sqrt{2}\|M\|\right)$. Hence for the sum we have: $\Gamma_{11}+\Gamma_{22}=\operatorname{trace}\left(C^{T} L C\right)=\operatorname{trace}\left(C C^{T} L\right)=\operatorname{trace}(L+M L)=\operatorname{trace}(L)+R$, where $|R|=\|L\| O_{P}\left(1 / n \rho_{n}\right)$. Also, for the product,

$$
\begin{aligned}
\Gamma_{11} \Gamma_{22} & =\operatorname{det}\left(C^{T} L C\right)=\operatorname{det}\left(C^{T} C\right) \operatorname{det}(L)=\operatorname{det}\left(I_{2 \times 2}-R^{T} R\right) \operatorname{det}(L) \\
& =\left(\left(1-\widehat{\mathbf{r}}_{1}^{T} \widehat{\mathbf{r}}_{1}\right)\left(1-\widehat{\mathbf{r}}_{2}^{T} \widehat{\mathbf{r}}_{2}\right)-\left(\widehat{\mathbf{r}}_{1}^{T} \widehat{\mathbf{r}}_{2}\right)^{2}\right) \operatorname{det}(L)=\operatorname{det}(L)(1+S),
\end{aligned}
$$

where $S$ is $O_{P}\left(1 / n \rho_{n}\right)$. Now, $\Gamma_{i i}$ for $i \in\{1,2\}$ are solutions to the quadratic equation $x^{2}-\left(L_{11}+L_{22}+R\right) x+L_{11} L_{22}(1+S)$. The solutions are given by

$$
\begin{aligned}
x & =\frac{L_{11}+L_{22}+R \pm \sqrt{\left(L_{11}+L_{22}+R\right)^{2}-4 L_{11} L_{22}(1+S)}}{2} \\
& =\frac{L_{11}+L_{22}+R \pm \sqrt{\left(L_{11}-L_{22}\right)^{2}+R^{\prime}}}{2} \quad \text { where }\left|R^{\prime}\right|=\|L\|^{2} O_{P}\left(1 / n \rho_{n}\right)
\end{aligned}
$$

If $L_{11}-L_{22}$ is the same order as $L_{i i}, i \in\{1,2\}$, then a little algebra yields the result. 
Lemma E.3. Let $R$ be defined as in Equation A.2. We have $\|R\|=$ $O_{P}\left(\frac{1}{\sqrt{n \rho_{n}}}\right)$.

Proof. In Equation A.3 we see that the two components are orthogonal in the sense that $(\widehat{Q}(\widehat{\Lambda} C-C \Lambda))^{T}(A R-R \Lambda)$ is the $2 \times 2$ zero matrix. Therefore by pre multiplying each side of Equation A.3 with its transpose we have

$Q^{T}(A-P)^{2} Q=(\widehat{Q}(\widehat{\Lambda} C-C \Lambda))^{T}(\widehat{Q}(\widehat{\Lambda} C-C \Lambda))+(A R-R \Lambda)^{T}(A R-R \Lambda)$

Hence via Weyl's identity and positive semi-definiteness of the first component on the R.H.S we have,

$$
\|(A-P) Q\|^{2} \geq\|A R-R \Lambda\|^{2}
$$

Since $\|R \Lambda\| \geq \lambda_{2}\|R\| / \sqrt{2}$, and $\|A R\| \leq \widehat{\lambda}_{3}\|R\|$, using Lemma A.6 (Equation A.7) we have $\|A R-R \Lambda\| \geq\left(\lambda_{2} / \sqrt{2}-\widehat{\lambda}_{3}\right)\|R\|=\lambda_{2} / \sqrt{2}\left(1+o_{P}(1)\right)\|R\|$. From Equation E.3 and Lemma A.6 (Equation A.7 shows that $\|(A-P) Q\|=$ $\left.O_{P}\left(\sqrt{n \rho_{n}}\right)\right)$ we get the following:

$$
\|R\| \leq \frac{\sqrt{2}\|(A-P) Q\|\left(1+o_{P}(1)\right)}{\lambda_{2}}=O_{P}\left(1 / \sqrt{n \rho_{n}}\right)
$$

Proof of Lemma 5.2. We have $Q^{T} A Q=C^{T} \widehat{\Lambda} C+R^{T} A R$. Lemma E.2 shows that $\left\|\operatorname{Eig}\left(C^{T} \widehat{\Lambda} C\right)-\widehat{\Lambda}\right\|=O_{P}(1)$. Using Corollary E.1 and Lemma 5.1 we see that $\hat{\lambda}_{1}, \hat{\lambda}_{2}, \widehat{\lambda}_{1}+\widehat{\lambda}_{2}$ and $\widehat{\lambda}_{1}-\widehat{\lambda}_{2}$ are of the form $C_{0} n \rho_{n}\left(1+O_{P}\left(\sqrt{\log n / n \rho_{n}}\right)\right.$, thus satisfying the condition of Lemma E.2. Using Lemma A.6 (Equation A.8) and Corollary E.1(Equation E.2), we have $\left\|R^{T} A R\right\| \leq\|R\|^{2}\left|\widehat{\lambda}_{3}\right|=O_{P}\left(\sqrt{\log n / n \rho_{n}}\right)$.

Thus for $i \in\{1,2\}$ we have:

$$
\left|\operatorname{Eig}_{i}\left(Q^{T} A Q\right)-\widehat{\Lambda}_{i i}\right| \leq\left|\operatorname{Eig}_{i}\left(C^{T} \widehat{\Lambda} C\right)-\widehat{\Lambda}_{i i}\right|+\left\|R^{T} A R\right\|=O_{P}(1) .
$$

On the other hand, since $Q^{T} A Q=Q^{T} P Q+Q^{T}(A-P) Q=\Lambda+Q^{T}(A-P) Q$, using Weyl's identity and Lemma A.6 (Equation A.5), we also have

$$
\text { For } i \in\{1,2\} \quad\left|\operatorname{Eig}_{i}\left(Q^{T} A Q\right)-\Lambda_{i i}\right|=O_{P}(1)
$$

The above two equations prove that for $i \in\{1,2\}, \widehat{\lambda}_{i}=\lambda_{i}+O_{P}(1)$.

Previously we have shown that $C$ is close to an unitary matrix; now we will prove that when $\gamma_{n}>0, C$ is actually close to the identity matrix. 
Proof of Lemma 5.3. Since $A \mathbf{v}_{i}=c_{i i} \widehat{\lambda}_{i} \widehat{\mathbf{v}}_{i}+A \widehat{\mathbf{r}}_{i}=\widehat{\lambda}_{i}\left(\mathbf{v}_{i}-\widehat{\mathbf{r}}_{i}\right)+A \widehat{\mathbf{r}}_{i}$,

$$
\text { For } i \in\{1,2\} \quad\left(A-\widehat{\lambda}_{i} I\right) \widehat{\mathbf{r}}_{i}=(A-P) \mathbf{v}_{i}+\left(\lambda_{i}-\widehat{\lambda}_{i}\right) \mathbf{v}_{i}
$$

Since $\widehat{\mathbf{r}}_{i}$ is orthogonal to $\widehat{\mathbf{v}}_{i}$, the R.H.S of the above equation is also orthogonal to $\widehat{\mathbf{v}}_{i}$. For definiteness, let $i=1$. We define the pseudo-inverse of $A-\widehat{\lambda}_{1} I$ as

$$
\left(A-\widehat{\lambda}_{1} I\right)^{+}:=\sum_{j \neq 1} \frac{\widehat{\mathbf{v}}_{j} \widehat{\mathbf{v}}_{j}^{T}}{\widehat{\lambda}_{j}-\widehat{\lambda}_{1}} ; \quad\left\|\left(A-\widehat{\lambda}_{1} I\right)^{+}\right\| \leq \frac{1}{\min _{j \neq 1}\left|\widehat{\lambda}_{j}-\widehat{\lambda}_{1}\right|} .
$$

Since $\left(A-\widehat{\lambda}_{1} I\right)^{+}\left(A-\widehat{\lambda}_{1} I\right)=I-\widehat{\mathbf{v}}_{1} \widehat{\mathbf{v}}_{1}^{T}$, and $\widehat{\mathbf{r}}_{1}^{T} \widehat{\mathbf{v}}_{1}=0$, left multiplying Equation E.4 by $\left(A-\widehat{\lambda}_{1} I\right)^{+}$we have:

$$
\widehat{\mathbf{r}}_{1}=\left(A-\widehat{\lambda}_{1} I\right)^{+}\left((A-P) \mathbf{v}_{1}+\left(\lambda_{1}-\widehat{\lambda}_{1}\right) \mathbf{v}_{1}\right)
$$

Using Lemma A.6 (Equation A.6)

$$
\left\|\widehat{\mathbf{r}}_{1}\right\| \leq \frac{1}{\min _{j \neq 1}\left|\widehat{\lambda}_{j}-\widehat{\lambda}_{1}\right|} O_{P}\left(\sqrt{n \rho_{n}}\right)
$$

For $j>2$, we have $\min _{j>2}\left|\widehat{\lambda_{1}}-\widehat{\lambda_{j}}\right| \geq\left|\widehat{\lambda}_{1}\right|-\max _{j>2}\left|\widehat{\lambda}_{j}\right| \geq C_{0} n \rho_{n}\left(1+o_{P}(1)\right)$. Also, $\left|\widehat{\lambda}_{1}-\widehat{\lambda}_{2}\right|=C_{0} n \rho_{n}\left(1+o_{P}(1)\right)$. Hence $1 / \min _{j \neq 1}\left|\widehat{\lambda}_{j}-\widehat{\lambda}_{1}\right|=O_{P}\left(1 / n \rho_{n}\right)$. This along with Equation E.5 gives the required bound on $\left\|\widehat{\mathbf{r}}_{1}\right\|$. An identical argument works for $\left\|\widehat{\mathbf{r}}_{2}\right\|$. Now, $c_{i i}^{2}=1-\left\|\widehat{\mathbf{r}}_{i}\right\|^{2}$ and hence we have:

$$
1-c_{i i}^{2}=O_{p}\left(1 / n \rho_{n}\right), \text { for } i \in\{1,2\} .
$$

Let us recall that $c_{21}=\mathbf{v}_{2}^{T} \widehat{\mathbf{v}}_{1}$. Now

$$
\mathbf{v}_{2}^{T}(A-P) \widehat{\mathbf{v}}_{1}=\widehat{\lambda}_{1} c_{21}-\lambda_{2} c_{21}=\left(\widehat{\lambda}_{1}-\lambda_{2}\right) c_{21}
$$

We also have: $\mathbf{v}_{2}^{T}(A-P) \widehat{\mathbf{v}}_{1}=\left(\mathbf{v}_{2}^{T}(A-P) \mathbf{v}_{1}-\mathbf{v}_{2}^{T}(A-P) \widehat{\mathbf{r}}_{1}\right) / c_{11}$. Now, $\mathbf{v}_{2}^{T}(A-P) \mathbf{v}_{1}$ is the off diagonal term of $Q^{T}(A-P) Q$, and from Lemma A.6 (Equation A.5), we can see that $\left|\mathbf{v}_{2}^{T}(A-P) \mathbf{v}_{1}\right| \leq\left\|Q^{T}(A-P) Q\right\|=O_{P}(1)$. Also, since $\left\|\widehat{\mathbf{r}}_{1}\right\|=O_{P}\left(1 / \sqrt{n \rho_{n}}\right)$, and $\left\|(A-P) \mathbf{v}_{2}\right\|=O_{P}\left(\sqrt{n \rho_{n}}\right)$ (Lemma A.6 Equation A.6), by the Cauchy-Schwarz inequality we have $\left|\mathbf{v}_{2}^{T}(A-P) \widehat{\mathbf{r}}_{1}\right|=$ $O_{P}(1)$. Since $1-c_{11}^{2}=O_{P}\left(1 / n \rho_{n}\right),\left|\mathbf{v}_{2}^{T}(A-P) \widehat{\mathbf{v}}_{1}\right|=O_{P}(1)$. We also have $\widehat{\lambda}_{1}=\lambda_{1}+O_{P}(1)$, and hence $\left(\widehat{\lambda}_{1}-\lambda_{2}\right) \sim\left(\lambda_{1}-\lambda_{2}\right)$. We can evaluate $\lambda_{1}-\lambda_{2}$ exactly as $C_{0} n \rho_{n}$, and hence the result.

$$
c_{21}=\mathbf{v}_{2}^{T}(A-P) \widehat{\mathbf{v}}_{1} /\left(\widehat{\lambda}_{1}-\lambda_{2}\right)=O_{P}\left(1 / n \rho_{n}\right)
$$

A similar argument yields the result for $c_{12}$. 
We now know that the norm of the residuals $\left(\widehat{\mathbf{r}}_{1}\right.$ and $\left.\widehat{\mathbf{r}}_{2}\right)$ are small. But in order to compute the distances in Equations 2.2 and 2.3, we would also need $\widehat{r}_{1}\left(C_{1}\right), \widehat{r}_{2}\left(C_{1}\right)$ etc. We now present the proof of Lemma 5.4.

Proof of Lemma 5.4. Let $\widehat{\mathbf{r}}_{1}=c_{12} \widehat{\mathbf{v}}_{2}+\mathbf{R}_{1}$, where $\mathbf{R}_{1}$ is orthogonal to both $\widehat{\mathbf{v}}_{1}$ and $\widehat{\mathbf{v}}_{2}$. Also $\left\|\mathbf{R}_{1}\right\| \leq\left\|\widehat{\mathbf{r}}_{1}\right\|=O_{P}\left(1 / n \rho_{n}\right)$. Hence, $\mathbf{v}_{2}^{T} \mathbf{R}_{1}=\widehat{\mathbf{r}}_{2}^{T} \mathbf{R}_{1}=$ $O_{P}\left(1 / n \rho_{n}\right)$ using the Cauchy-Schwarz inequality.

Because, $\widehat{\mathbf{r}}_{1}$ is orthogonal to $\widehat{\mathbf{v}}_{1}, \mathbf{v}_{1}^{T} \widehat{\mathbf{r}}_{1}=\widehat{\mathbf{r}}_{1}^{T} \widehat{\mathbf{r}}_{1}=O_{P}\left(1 / n \rho_{n}\right)$. Also, $\mathbf{v}_{2}^{T} \widehat{\mathbf{r}}_{1}=$ $\mathbf{v}_{2}^{T}\left(c_{12} \widehat{\mathbf{v}}_{2}+\mathbf{R}_{1}\right)=c_{12} c_{22}+\widehat{\mathbf{r}}_{2}^{T} \mathbf{R}_{1}$. Lemma 5.3 shows that $c_{12}=O_{P}\left(1 / n \rho_{n}\right)$, and hence $\mathbf{v}_{2}^{T} \widehat{\mathbf{r}}_{1}$ is $O_{P}\left(1 / n \rho_{n}\right)$.

$$
\begin{aligned}
& n \pi x_{1} \widehat{r}_{1}\left(C_{1}\right)+n(1-\pi) y_{1} \widehat{r}_{1}\left(C_{2}\right)=\widehat{\mathbf{r}}_{1}^{T} \widehat{\mathbf{r}}_{1}=: W_{1} \\
& n \pi x_{2} \widehat{r}_{1}\left(C_{1}\right)+n(1-\pi) y_{2} \widehat{r}_{1}\left(C_{2}\right)=\mathbf{v}_{2}^{T} \widehat{\mathbf{r}}_{1}=: W_{2}
\end{aligned}
$$

Solving for $\widehat{r}_{1}\left(C_{1}\right)$ and $\widehat{r}_{1}\left(C_{2}\right)$ we see that, $\widehat{r}_{1}\left(C_{1}\right)=\frac{y_{2} W_{1}-y_{1} W_{2}}{\left(x_{1} y_{2}-x_{2} y_{1}\right) n \pi}$, and $\widehat{r}_{1}\left(C_{2}\right)=\frac{x_{2} W_{1}-x_{1} W_{2}}{\left(x_{2} y_{1}-x_{1} y_{2}\right) n(1-\pi)}$. Using Lemma 5.1 we see that $x_{1} y_{2}-x_{2} y_{1} \neq 0$ for $\gamma_{n}>0$. In particular, it is of the form $C_{0} / n$. Hence we have:

$$
\widehat{r}_{1}\left(C_{1}\right)=O_{p}\left(1 / n^{3 / 2} \rho_{n}\right) \quad \widehat{r}_{1}\left(C_{2}\right)=O_{p}\left(1 / n^{3 / 2} \rho_{n}\right)
$$

The result for $\widehat{r}_{2}\left(C_{1}\right)$ and $\widehat{r}_{2}\left(C_{2}\right)$ can be obtained analogously.

\section{F. Supplementary material for analysis of the normalized method.}

F.1. Population eigenvalues and eigenvectors. We start with the proof of Lemma 6.1, which summarizes the population eigenvalues and eigenvectors under normalization.

Proof of Lemma 6.1. Let the eigenvalues of $\widetilde{P}$ be $\nu_{1}, \nu_{2}$, etc. in decreasing order of absolute value. Using an argument analogous to the first part of the proof of Lemma 5.1 we see that $\nu_{i}=O(1 / n)$ for $i>2$.

Note that for $i \neq j, \widetilde{P}(i, j)$ equals $\alpha_{n} / n \mu_{1}$ when $i, j \in C_{1}, \beta_{n} / n \mu_{2}$ when $i, j \in C_{2}$, and $\gamma_{n} / n \sqrt{\mu_{1} \mu_{2}}$ when $i \in C_{1}, j \in C_{2}$. Let us consider the blockwise constant matrix $\widetilde{P}_{B}$, such that $\forall i \neq j \widetilde{P}_{B}(i, j)=\widetilde{P}(i, j)$, for $i \in C_{1}$ $\widetilde{P}_{B}(i, i)=\alpha_{n} / n \mu_{1}$ and for $i \in C_{2}, \widetilde{P}_{B}(i, i)=\beta_{n} / n \mu_{2}$. When $\alpha_{n} \beta_{n} \neq \gamma_{n}^{2}$, $\widetilde{P}_{B}$ is rank two, where the principal eigenvalues are constants w.r.t $n$. Since $\left\|\widetilde{P}-\widetilde{P}_{B}\right\|=O(1 / n)$, a simple application of Weyl's inequality shows that $\nu_{1}, \nu_{2}$ are constants w.r.t $n$, whereas all the rest $\underset{\widetilde{P}}{ } O(1 / n)$.

It is well known that the largest eigenvalue of $\widetilde{P}$ is one. Using the PerronFrobenius theorem, we see that for $\gamma_{n}>0$, there is only one eigenvalue equal to one. The first eigenvector has entries proportional to the square root of 
the corresponding row sum of the matrix $P$. For the second eigenvector, we construct a vector orthogonal to the first eigenvector and see that it is an eigenvector with eigenvalue $1-\gamma_{n} \mu / \mu_{1} \mu_{2}$. This must be the second largest eigenvalue since we have shown that $\widetilde{\nu}_{i}=O(1 / n)$ for $i>2$. It can be shown that $\nu_{2}=-1$ iff $\alpha_{n}=0, \beta_{n}=0, \gamma_{n}>0$. The condition $\alpha_{n} \beta_{n} \neq \gamma_{n}^{2}$ ensures that $\nu_{2}$ is bounded away from zero by a constant.

F.2. Our approximation of the second eigenvector of $\widetilde{A}$. Let us recall that we approximate $\widetilde{\mathbf{u}}_{2}$ in two steps. First we construct a guess $\mathbf{u}_{g}^{0}$ orthogonal to $\widetilde{\mathbf{u}}_{1}$ and then take a power iteration step from it to compute the second guess $\mathbf{u}_{g}$. We now prove Lemma 6.2, which computes term by term Taylor expansion of $\widetilde{A} \mathbf{u}_{g}^{0}$. This will be used later in the proof of Lemma 6.3, which bounds the error of $\mathbf{u}_{g}$.

Proof of Lemma 6.2. We have $\left(\widetilde{A} \mathbf{u}_{g}^{0}\right)_{i}=\frac{d_{i}^{(1)}}{\sqrt{d_{i} E_{1}}}-\frac{d_{i}^{(2)}}{\sqrt{d_{i}} E_{2}}$. We recall that for $i \in C_{1}, E\left[d_{i}\right]=n \mu_{1}$, and for $i \in C_{2}, E\left[d_{i}\right]=n \mu_{2}$. In the following Taylor series expansions for $i \in C_{1}, S_{1}$ and $R_{i}$ are the remainder terms.

$$
\begin{aligned}
& \frac{1}{E_{1}}=\frac{1}{n^{2} \pi \mu_{1}}\left(1-\frac{E_{1}-n^{2} \pi \mu_{1}}{n^{2} \pi \mu_{1}}\left(1+S_{1}\right)\right) \\
& \frac{1}{\sqrt{d_{i}}}=\frac{1}{\sqrt{n \mu_{1}}}\left(1-\frac{d_{i}-n \mu_{1}}{2 n \mu_{1}}\left(1+R_{i}\right)\right),
\end{aligned}
$$

Simple applications of the Chernoff bound (Lemmas A.2 and A.4) show that $\max _{i}\left|R_{i}\right|=o_{P}(1)$ and $|S|=o_{P}(1)$. Let $\bar{X}:=X-E[X]$. Thus for $i \in C_{1}$, and $T_{i}=T_{i}^{(1)}+T_{i}^{(2)}+T_{i}^{(3)}$,

$$
\begin{aligned}
\frac{d_{i}^{(1)}}{\sqrt{d_{i}} E_{1}} & =\frac{d_{i}^{(1)}}{\sqrt{n \mu_{1}}} \frac{1}{n^{2} \pi \mu_{1}}(1-\frac{\bar{d}_{i}}{2 n \mu_{1}}-\underbrace{\frac{\bar{d}_{i} R_{i}}{2 n \mu_{1}}}_{T_{i}^{(1)}} \underbrace{-\frac{\bar{E}_{1}\left(1+S_{1}\right)}{n^{2} \pi \mu_{1}}}_{T_{i}^{(2)}} \underbrace{+\frac{\bar{d}_{i}\left(1+R_{i}\right)}{2 n \mu_{1}} \frac{\bar{E}_{1}\left(1+S_{1}\right)}{n^{2} \pi \mu_{1}}}_{T_{i}^{(3)}}) \\
& =\frac{(n \pi-1) \alpha_{n}}{\sqrt{n \mu_{1}}} \frac{1}{n^{2} \pi \mu_{1}}(1+\underbrace{\frac{\bar{d}_{i}^{(1)}}{(n \pi-1) \alpha_{n}}}_{G_{i}}-\underbrace{\frac{\bar{d}_{i}}{2 n \mu_{1}}}_{H_{i}}+T_{i}^{\prime}) \\
T_{i}^{\prime} & =T_{i}+T_{i} G_{i}-G_{i} H_{i}
\end{aligned}
$$

We note that $G_{i}-H_{i}$ is of the form $c_{1} \frac{\bar{d}_{i}^{(1)}}{n \rho_{n}}-c_{2} \frac{\bar{d}_{i}^{(2)}}{n \rho_{n}}$ for constants $c_{1}, c_{2}$, and using an argument similar to Lemma 5.5 (Equation 5.4) we see that $\|G-H\|$ 
as well as $\|G\|$ and $\|H\|$ are of the form $C_{0} / \sqrt{\rho_{n}}\left(1+o_{P}(1)\right)$. We will show that $\left\|T^{\prime}\right\|$ is $o_{P}\left(1 / \sqrt{\rho_{n}}\right)$.

First we note that using applications of Chernoff bound (details in Lemmas A.2, A.4), we have:

$$
\begin{aligned}
\max _{i} R_{i} & =o_{P}(1) ; & \left\|T^{(1)}\right\| & =\|H\| o_{P}(1) \\
\left\|T^{(2)}\right\| & =O_{P}\left(\frac{1}{n}\right) ; & \left\|T^{(3)}\right\| & =\|H\| o_{P}(1)
\end{aligned}
$$

Since, $\|H\|$ and $\|G-H\|$ are of the same order, we have $\|T\|=\|G-H\| o_{P}(1)$. For the $G_{i} H_{i}$ term, since $\bar{d}_{i}=d_{i}^{(1)}+d_{i}^{(2)}$, this can be written as:

$$
G_{i} H_{i}=\frac{\left(\bar{d}_{i}^{(1)}\right)^{2}}{2 n \mu_{1}(n \pi-1) \alpha_{n}}+\frac{\bar{d}_{i}^{(1)} \bar{d}_{i}^{(2)}}{2 n \mu_{1}(n \pi-1) \alpha_{n}} .
$$

Using Lemma A.5 we see that the vectors formed by the two summands on the R.H.S of the above equation are of norm $O_{P}\left(1 / n \rho_{n}^{2}\right)$. Hence using the Cauchy-Schwarz inequality one can bound the norm of $\left\langle G_{i} H_{i}\right\rangle$ by $O_{P}\left(1 / \sqrt{n \rho_{n}^{2}}\right)$, which is again $\|G-H\| o_{P}(1)$. Finally for $Y_{i}$, we have

$$
T_{i} G_{i}=G_{i} H_{i} R_{i}+G_{i} T_{i}^{(2)}+G_{i} H_{i}\left(1+R_{i}\right) T_{i}^{(2)}
$$

Using Equation F.1 and the bound on $\left\langle G_{i} H_{i}\right\rangle$ we have $\|Y\|=o_{P}(G-H)$.

Similarly we can show that:

$$
\begin{aligned}
\frac{d_{i}^{(2)}}{\sqrt{d_{i}} E_{2}} & =\frac{\gamma_{n}}{\sqrt{n \mu_{1}}} \frac{1}{n \mu_{2}}\left(1-\frac{\bar{d}_{i}}{2 n \mu_{1}}+\frac{\bar{d}_{i}^{(2)}}{n(1-\pi) \gamma_{n}}+T_{i}^{\prime \prime}\right) \\
\left\|T_{i}^{\prime \prime}\right\| & =o_{P}\left\|\left\langle-\bar{d}_{i} / 2 n \mu_{1}+\bar{d}_{i}^{(2)} / n(1-\pi) \gamma_{n}\right\rangle\right\|=o_{P}\left(1 / \sqrt{\rho_{n}}\right)
\end{aligned}
$$

Thus, for the $i^{t h}$ entry of $\widetilde{A} \mathbf{u}_{g}^{0}$ we have:

$$
\begin{aligned}
& {\left[\widetilde{A} \mathbf{u}_{g}^{0}\right]_{i}= \begin{cases}\frac{\nu_{2}}{n \pi \sqrt{n \mu_{1}}}\left(1-\frac{\bar{d}_{i}}{2 n \mu_{1}}+\frac{\bar{d}_{i}^{(1)}}{n \mu_{1} \nu_{2}}-\frac{\bar{d}_{i}^{(2)}}{n \mu_{2} \nu_{2}} \frac{\pi}{1-\pi}+M_{i}\right) & i \in C_{1} \\
-\frac{\nu_{2}}{n(1-\pi) \sqrt{n \mu_{2}}}\left(1-\frac{\bar{d}_{i}}{2 n \mu_{2}}-\frac{\bar{d}_{i}^{(1)}}{n \mu_{1} \nu_{2}} \frac{1-\pi}{\pi}+\frac{\bar{d}_{i}^{(2)}}{n \mu_{2} \nu_{2}}+M_{i}^{\prime}\right) & i \in C_{2}\end{cases} } \\
& \|M\|,\left\|M^{\prime}\right\|=o_{P}\left(1 / \sqrt{\rho_{n}}\right) \\
& \left\|\widetilde{A} \mathbf{u}_{g}^{0}\right\| \sim \nu_{2} \sqrt{\frac{\mu}{n^{2} \pi(1-\pi) \mu_{1} \mu_{2}}}
\end{aligned}
$$


Proof of Lemma 6.3. We have:

$$
\sqrt{d_{i}}=\sqrt{n \mu_{1}}+\frac{\bar{d}_{i}\left(1+S_{i}\right)}{2 \sqrt{n \mu_{1}}} ; \quad \frac{1}{E_{1}}=\frac{1}{n^{2} \pi \mu_{1}}\left(1-\frac{E_{1}-n^{2} \pi \mu_{1}}{n^{2} \pi \mu_{1}}\left(1+S_{1}\right)\right),
$$

Using arguments similar to Lemma 4.3 one can show that $\max _{i}\left|S_{i}\right|$ and $\left|S_{1}\right|=o_{P}(1)$. This gives the following entrywise Taylor expansion for $\mathbf{u}_{g}^{0}$.

$$
\begin{aligned}
u_{g}^{0}(i) & =\left\{\begin{array}{lr}
\frac{1}{n \pi \sqrt{n \mu_{1}}}\left(1+\frac{\bar{d}_{i}}{2 n \mu_{1}}+T_{i}\right) & i \in C_{1} \\
\frac{-1}{n(1-\pi) \sqrt{n \mu_{2}}}\left(1+\frac{\bar{d}_{i}}{2 n \mu_{2}}+T_{i}^{\prime}\right) & i \in C_{2}
\end{array}\right. \\
\|T\|,\left\|T^{\prime}\right\|=o_{P}\left(1 / \sqrt{\rho_{n}}\right) &
\end{aligned}
$$

It immediately follows that

$$
\left\|\mathbf{u}_{g}^{0}\right\|^{2} \sim \frac{\mu}{n^{2} \pi(1-\pi) \mu_{1} \mu_{2}} .
$$

Now we represent $\mathbf{u}_{g}^{0}$ as

$$
\mathbf{u}_{g}^{0} /\left\|\mathbf{u}_{g}^{0}\right\|=c_{g}^{0} \widetilde{\mathbf{u}}_{2}+\mathbf{r}_{g}^{0}
$$

where $c_{g}^{0}=\widetilde{\mathbf{u}}_{2}^{T} \mathbf{u}_{g}^{0} /\left\|\mathbf{u}_{g}^{0}\right\|$. Since $\mathbf{u}_{g}^{0}$ is orthogonal to $\widetilde{\mathbf{u}}_{1}$ (by construction), and $\widetilde{\mathbf{u}}_{2}$ is orthogonal to $\widetilde{\mathbf{u}}_{1}$ by definition, $\mathbf{r}_{g}^{0}$ is orthogonal to both $\widetilde{\mathbf{u}}_{1}$ and $\widetilde{\mathbf{u}}_{2}$.

$$
\frac{\widetilde{A} \mathbf{u}_{g}^{0}-\nu_{2} \mathbf{u}_{g}^{0}}{\left\|\mathbf{u}_{g}^{0}\right\|}=c_{g}^{0} \widetilde{\mathbf{u}}_{2}\left(\widetilde{\nu}_{2}-\nu_{2}\right)+\left(\widetilde{A}-\nu_{2} I\right) \mathbf{r}_{g}^{0}
$$

Now taking the squared norm of both sides yields,

$$
\frac{\left\|\widetilde{A} \mathbf{u}_{g}^{0}-\nu_{2} \mathbf{u}_{g}^{0}\right\|^{2}}{\left\|\mathbf{u}_{g}^{0}\right\|^{2}} \geq\left\|\left(\widetilde{A}-\nu_{2} I\right) \mathbf{r}_{g}^{0}\right\|^{2} \geq\left(\nu_{2}-\widetilde{\nu}_{3}\right)^{2}\left\|\mathbf{r}_{g}^{0}\right\|^{2}
$$

since $\left\|\left(\widetilde{A}-\nu_{2} I\right) \mathbf{r}_{g}^{0}\right\| \geq\left\|\nu_{2} \mathbf{r}_{g}^{0}\right\|-\left\|\widetilde{A} \mathbf{r}_{g}^{0}\right\| \geq\left(\nu_{2}-\widetilde{\nu}_{3}\right)\left\|\mathbf{r}_{g}^{0}\right\|$. Since $\widetilde{\nu}_{3}$ the third largest eigenvalue of $\widetilde{A}$ in magnitude, we have $\left\|\widetilde{A} \mathbf{r}_{g}^{0}\right\| \leq \widetilde{\nu}_{3}\left\|\mathbf{r}_{g}^{0}\right\|$, since $\mathbf{r}_{g}^{0}$ is orthogonal to both the first and second eigenvectors of $\widetilde{A}$.

In order to compute $\left\|\widetilde{A} \mathbf{u}_{g}^{0}-\nu_{2} \mathbf{u}_{g}^{0}\right\|^{2}$, we note that the leading term vanishes, and the first order term dominates the remainder term. Now using Lemma 6.2 and Equation 5.4, for some deterministic constants $\tau_{k, 1}$ and $\tau_{k, 2}(k \in\{1,2\})$ (which are non-zero for $\gamma_{n}>0$ ), we have:

$$
\left\|\widetilde{A} \mathbf{u}_{g}^{0}-\nu_{2} \mathbf{u}_{g}^{0}\right\|^{2} \sim \frac{\nu_{2}^{2}}{n^{3} \rho_{n}} \sum_{k=1}^{2} \sum_{i \in C_{k}}\left(\tau_{k, 1} \frac{\bar{d}_{i}^{(1)}}{n \mu_{1}}+\tau_{k, 2} \frac{\bar{d}_{i}^{(2)}}{n \mu_{2}}\right)^{2}=O_{P}\left(\frac{1}{n^{3} \rho_{n}^{2}}\right)
$$


Using Theorem 2.1, Lemma 6.1, and an argument identical to that in Corollary E.1 we know that $\widetilde{\nu}_{3}=O_{P}\left(\sqrt{\log n / n \rho_{n}}\right)$ and hence $\left|\nu_{2}-\widetilde{\nu}_{3}\right| \sim \nu_{2}$, where $\nu_{2}$ is a deterministic constant independent of $n$. Plugging these into equation F.5, and using Equation F.3 we have:

$$
\left(\nu_{2}-\widetilde{\nu}_{3}\right)^{2}\left\|\mathbf{r}_{g}^{0}\right\|^{2}=O_{P}\left(\frac{1}{n \rho_{n}}\right) \rightarrow\left\|\mathbf{r}_{g}^{0}\right\|^{2}=O_{P}\left(\frac{1}{\sqrt{n \rho_{n}}}\right)
$$

From equation F.4 we have the following ortho-normal decomposition of $\mathbf{u}_{g}$ :

$$
\mathbf{u}_{g}=\frac{c_{g}^{0} \widetilde{\nu}_{2}}{\left\|\widetilde{A} \mathbf{u}_{g}^{0}\right\| /\left\|\mathbf{u}_{g}^{0}\right\|} \widetilde{\mathbf{u}}_{2}+\frac{\widetilde{A} \mathbf{r}_{g}^{0}}{\left\|\widetilde{A} \mathbf{u}_{g}^{0}\right\| /\left\|\mathbf{u}_{g}^{0}\right\|}=c_{g} \widetilde{\mathbf{u}}_{2}+\mathbf{r}_{g}
$$

where $c_{g}:=\left(\widetilde{\mathbf{u}}_{2}\right)^{T} u_{g}$ and $\mathbf{r}_{g}:=\frac{\widetilde{A} \mathbf{r}_{g}^{0}}{\left\|\widetilde{A} \mathbf{u}_{g}^{0}\right\| /\left\|\mathbf{u}_{g}^{0}\right\|}$. From Lemma 6.2 we have $\left\|A u_{g}^{0}\right\| \sim\left\|\nu_{2} \mathbf{u}_{g}^{0}\right\|$. This shows that the residual $\mathbf{r}_{g}$ has norm of the following order:

$$
\left\|\mathbf{r}_{g}\right\|=\frac{\left\|\widetilde{A} \mathbf{r}_{g}^{0}\right\|}{\left\|\widetilde{A} \mathbf{u}_{g}^{0}\right\| /\left\|\mathbf{u}_{g}^{0}\right\|}=O_{P}\left(\frac{\widetilde{\nu}_{3}\left\|\mathbf{r}_{g}^{0}\right\|}{\left\|\widetilde{A} \mathbf{u}_{g}^{0}\right\| /\left\|\mathbf{u}_{g}^{0}\right\|}\right)=O_{P}\left(\sqrt{\frac{\log n}{n^{2} \rho_{n}^{2}}}\right)
$$

But $c_{g}^{2}=1-\left\|\mathbf{r}_{g}\right\|^{2}=1-o_{P}(1)$.

\section{References.}

[1] Oliveira, R. I. (2009). Concentration of the adjacency matrix and of the Laplacian in random graphs with independent edges. Preprint.

[2] Rohe, K., Chatterjee, S. and Yu, B. (2011). Spectral clustering and the highdimensional stochastic blockmodel. Annals of Statistics 39 1878-1915.

[3] Sarkar, P. and Bickel, P. J. (2014). Role of Normalization in Spectral Clustering for Stochastic Blockmodels. 